Exhibits concerned with research techniques included the compact lightweight McArthur microscope, designed for field-work ; new uses for cellulose acetate in the culture of anaerobic bacteria and in electrophoresis (W. H. Pierce); and the confining of insects to treated plants under controlled conditions, for use in insecticide screening (D. T. Saggers).
There were many other exhibits, of no less interest and attractiveness than those mentioned above, and the exhibits as a whole provided starting-points for the informal discussion and conversation between those present which are, after all, the prime purpose of a conversazione.

\title{
MARIAN SMOLUCHOWSKI
}

$\mathrm{M}$ ARIAN SMOLUCHOWSKI, the great Polish theoretical physicist, died during the First World War on September 5, 1917, in Cracow, and a description of his life and work was published by $\mathrm{A}$. Teske three years ago ("Marian Smoluchowski : His Life and Work". Polish State Scientific Publishing House, Cracow, 1955). However, for those not familiar with the Polish language, the brief account of Smoluchowski's career and of his contributions to the theory of probabilities in physics given in the article contributed by $\mathrm{S}$. Ulam to the October number of The American Journal of Physics $(25,475 ; 1957)$ will be very welcome.

Smoluchowski was born in Vienna in 1872. His father was a Cracow lawyer who later became secretary of the Chancellery at the Court of the Emperor Francis Joseph. His mother, too, was well educated, and Smoluchowski was brought up in highly intellectual surroundings. He early showed an interest in science and he studied mathematics and physics at the University of Vienna, attending the lectures given by Boltzmann, Mach and others. He published his first scientific paper in his second year at the University, finally graduating with the highest honours. After a year in Paris working with Lipmann, he studied for a year in Glasgow under Kelvin and then spent a few months in the laboratory of Prof. Warburg in Charlottenberg, finally returning to Vienna to become a lecturer in the University of that city. In 1899 he became a lecturer in Lwow, and the next year, at twenty-eight, he was promoted to a chair of theoretical physies, thus becoming the youngest professor in Austria. In 1913 he was offered, and accepted, the chair of experimental physics at Cracow, but was evacuated to Vienna in the early part of the First World War. The University of Cracow made him rector when he was invited again in 1917 to the chair of theoretical physics in Vienna, but Smoluchowski died suddenly at the age of forty. five before he could deliver his inaugural address entitled "On the Uniformity of Laws of Nature".

Probability theory played an important part in physics during the forty years following Smoluchowski's premature death, and Smoluchowski can claim to have started and anticipated much of the subsequent development. He, together with and independently of Einstein, elaborated and carried forward the ideas of Maxwell and Boltzmann. One of Smoluchowski's mathematical achievements was the clarification of the ergodic hypothesis of Boltzmann and of its place and meaning in statistical mechanics. He realized the difference between the logical structure of the Maxwell and Boltzmann theories and the statistical mechanics of Gibbs. Though primarily a theoretical physicist, Smoluchowski was also very adept in the design and construction of instruments and an extremely good glass-blower. While at Lwow he carried out a brilliant series of experiments confirming Lord Rayleigh's theory that the blue of the sky was caused by the scattering of light. He paid several visits to Great Britain both for mountaineering in Scotland (one of his great passions), and also on academic business. He was an active member of many scientific societies, in particular the Polish Physical Society, of which he became the first president, and he contributed many papers to scientific periodicals, some collections of which have been published since his death.

\section{MENTAL HEALTH RESEARCH FUND}

$\mathrm{O}$ FFICIAL figures show that in 1956 there were in England and Wales more than 204,000 hospital beds occupied by patients suffering from mental illness or mental deficiency. This figure can be com. pared with the 219,000 occupied beds for all other diseases put together. In addition, there were more than 81,000 mental defectives under some sort of supervision provided by the local authority and an unknown number of persons suffering from neuroses which impair their efficiency and happiness. This problem is increasing. In 1949, it led to the formation of the Mental Health Research Fund by a small group of scientists, doctors, and laymen who shared the belief that mental health and mental illness were not receiving that share of attention in research which their importance demands.

The Fund was registered in 1952 and heads of leading research institutions in Great Britain were invited to say in what ways they would wish to use any money that might be available for work in promoting mental health.

A conference at $O x f o r d$ was then convened to which were invited a wide cross-section of scientists, ranging from anatomists to sociologists, as well as a number of practising psychiatrists and professors of psychiatry. Its terms of reference were to answer the following questions: What are the ignorances which to-day principally hamper understanding of the nature, prevention and cure of mental illness? What advances in research are most likely to remove these and so help to reduce the population of mental hospitals and institutions for delinquents?

The Fund has now defined its main purpose and responsibilities as follows: The establishment of research fellowships in the several fields of mental 